Philosophie ANTIQUE
Philosophie antique

Problèmes, Renaissances, Usages

14 | 2014

Le devoir

\title{
Voula tsouna, Philodemus, On Property Management
}

\section{Annick Monet}

\section{OpenEdition \\ Journals}

Édition électronique

URL : https://journals.openedition.org/philosant/858

DOI : 10.4000/philosant.858

ISSN : 2648-2789

Éditeur

Éditions Vrin

\section{Édition imprimée}

Date de publication : 1 novembre 2014

Pagination : 352-355

ISBN : 978-2-7574-0855-1

ISSN : $1634-4561$

\section{Référence électronique}

Annick Monet, «Voula tsouna, Philodemus, On Property Management », Philosophie antique [En ligne], 14। 2014, mis en ligne le 01 novembre 2018, consulté le 03 décembre 2022. URL : http:// journals.openedition.org/philosant/858; DOI : https://doi.org/10.4000/philosant.858

\section{(c) $\underset{\mathrm{EY}}{(\mathrm{NQ})} \mathrm{\Theta O}$}

Creative Commons - Attribution - Pas d'Utilisation Commerciale - Pas de Modification 4.0 International - CC BY-NC-ND 4.0

https://creativecommons.org/licenses/by-nc-nd/4.0/ 
struire de larges séquences historico-conceptuelles touchant des questions aussi diverses que les apories du mouvement, les conceptions continuistes et discontinuistes de l'espace et du temps, la problématique physique et géométrique des lignes indivisibles, les rapports entre les idées d'angle et de minimum, etc.

Ce foisonnement, avec la profusion de notes, le caractère digressif de certains développements, quelques redondances, qui en sont autant de conséquences, peut rendre difficile la lecture en continu de l'ouvrage. Celui-ci n'en constitue pas moins pour le chercheur et même simplement pour le lecteur intéressé à l'histoire de la philosophie et de la science de l'Antiquité une ressource exceptionnelle.

Alain GIGANDET

Université Paris Est Créteil-Paris 12

Voula Tsouna, Philodemus, On Property Management, Atlanta, Society of Biblical Literature, 2012 (Writings from the Greco-Roman World, 33), xlv + 125 pages, ISBN 978-1-58983-667-9.

Sur le modèle du On Death $I V$ (De morte) de Philodème, publié chez le même éditeur en 2009 par W. B. Henry, cet ouvrage propose, après une introduction de trente-quatre pages, une traduction - fondée sur l'édition donnée par Christian Jensen en 1906 pour Teubner - de cet écrit de Philodème tenu par lui comme un livre sur l'économie. Même si dans les « Acknowledgements » (p. vii) V. Tsouna rappelle qu'elle a participé à la traduction de L'Économie pour Les Épicuriens coédité par Daniel Delattre et Jackie Pigeaud (Paris, Gallimard, 2010, Bibliothèque de la Pléiade), il n'est guère fait mention des nouvelles lectures et conjectures dont cette publication a été l'occasion et qui sont disponibles en ligne sur le site du CISPE (http://www.herculanensiacispe.org/langue-francaise.html), à l'exception des pages 72 et 95 (une mention en note 42 , mais pas en note 44).

Ce livre de Philodème appartient à un ensemble plus vaste consacré aux vices et aux vertus opposées et composé d'au moins dix rouleaux-livres. Le titre donné à la fin du PHerc. 1424 est le plus complet de ceux qui sont conservés : Sur les vices et des vertus qui leur sont opposées, des personnes chez qui ils sont présents et des situations dans lesquelles on les rencontre, livre IX (ce qu'on pourrait aussi traduire: Des vices et des vertus qui leur sont opposées, des hommes chez qui ils se rencontrent et des domaines qu'ils concernent; toutefois, aucun sous-titre mentionnant explicitement le domaine de l'économie n'y figure, et le titre qui désigne aujourd'hui couramment l'ouvrage, Peri oikonomias, est simplement déduit du contenu. De fait, le terme grec oikovouia (oikonomia), traduit en anglais américain par property management (《gestion d'une propriété »), y est récurrent, et fait, à juste titre, l'objet d'une explication dès le début de l'introduction (p.xi-xii).

L'introduction, qui reprend dans les grandes lignes le contenu développé par Philodème, sans toujours suivre de façon linéaire la progression du livre, développe cinq points : les thèses de Xénophon et de Théophraste, leurs points communs; la critique qu'en fait Philodème; la position épicurienne en matière d'acquisition et de conservation des biens; la justification de la gestion d'une propriété par une personne qui prétend à une vie conforme à la doctrine épicurienne; le philosophe épicurien comme administrateur ; les sources de revenu. 
Au premier chapitre de l'introduction, V. Tsouna rappelle les deux sources qui faisaient autorité dans l'antiquité en matière de gestion d'une propriété, Xénophon et Théophraste, et leurs points communs : ce sont les auteurs retenus par Philodème dans son livre. V. Tsouna résume les thèses de L'Économique de Xénophon puis des Économiques de Théophraste (écrit qui lui est attribué explicitement par Philodème en col. VII) qui sont discutées dans le texte de Philodème : l'économie définie comme techne, les quatre spécialités de l'expert en économie (acquisition, conservation, arrangement et usage des richesses), et la nécessité de faire des profits.

La deuxième partie de l'introduction rend compte des critiques que Philodème adresse à Xénophon et à Théophraste, dont il a d'abord résumé, successivement, les thèses sous forme de montages de citations. V. Tsouna souligne en effet, dans les notes finales de la traduction, que les toutes premières colonnes conservées de cette fin de rouleau constituent une paraphrase de l'écrit de Théophraste et pas encore la reprise critique que peut en faire Philodème. D'ailleurs, alors que dans le volume Les Épicuriens (p. 595), D. Delattre et V. Tsouna font précéder les colonnes A et B du titre «Fin du résumé de "L'Économique" de Théophraste », on se demande pourquoi l'introduction omet de mentionner cette manière de procéder typiquement philodémienne. V. Tsouna détaille les objections faites à Socrate sur diverses notions : richesse, profit, possession, pauvre, riche, maîtres et esclaves. Les différences principales entre la position épicurienne, d'un côté, et celles de Xénophon et de Théophraste, de l'autre, portent sur le rôle de la femme, les rapports du maître avec les serviteurs et les esclaves, et les activités propres à un administrateur. Ce que Philodème critique surtout, c'est que bon nombre des conseils donnés par Xénophon et repris par Théophraste ne sont pas compatibles avec la « vie heureuse $\gg$, ce vers quoi tend précisément le philosophe.

La position de Philodème sur la gestion de la propriété (col. XII-XXIV) est explicitée dans le troisième chapitre de l'introduction. Le bon administrateur n'est ni un profane dans le domaine de l'économie, ni un expert (comme peut l'être Ischomaque dans l'Économique de Xénophon), mais un administrateur philosophe qui veut se conformer au $\kappa \alpha \lambda \tilde{\omega} \zeta \zeta \tilde{\eta} \nu$ (le « vivre bien »). On notera que cette expression, importante dans la pensée épicurienne, se trouve traduite de deux façons différentes : « to live well » (aux col. XII et XVI) et «live decorously » (col. XIII). En outre, à l'adverbe restitué dexios (col. XIII) et traduit par «live competently », on préférera hedeos, conjecture adoptée dans le volume des Épicuriens (p. 605), qui renvoie à la notion de « vivre plaisamment » critiquée par Plutarque. Loin de se résigner à vivre dans la pauvreté pour être libre de tout souci, le sage épicurien administre sa propriété en obéissant aux priorités philosophiques. Philodème se démarque ainsi non seulement de Xénophon et de Théophraste (en se limitant aux tâches économiques, à l'acquisition et à la conservation des richesses), mais aussi des Cyniques et rappelle le débat qui les opposa jadis à Métrodore de Lampsaque. La conception épicurienne de l'oikonomia est fondée sur deux notions fondamentales : le calcul des plaisirs et des peines et la mesure (metron) de la richesse. Si les Épicuriens et les Cyniques considèrent que la meilleure vie consiste à s'éviter toute peine et inquiétude, ils divergent sur la façon d'y parvenir. V. Tsouna ne manque pas de souligner que Philodème s'inscrit bien dans la pensée d'Épicure: 
possession de richesses et administration de biens ne vont pas à l'encontre des principes épicuriens.

D'aucuns pourraient penser que l'administrateur épicurien n'est pas un bon administrateur. Rien de tel : c'est ce que s'emploie à montrer la quatrième partie de l'introduction qui reprend les principaux arguments de Philodème. L'administrateur épicurien ne cherche pas à accroître ses richesses, et la préconception (prolepsis) du «bon financier » désigne tout naturellement le sage; enfin, il convient de distinguer l'expert en administration de biens du philosophe, qui n'en possède pas la techne.

Le cinquième et dernier chapitre de l'introduction se concentre sur les colonnes XXII et XXIII qui traitent des sources de revenu. Ce passage rend compte de la discussion entre Philodème et des adversaires non identifiés à propos de la tranquillité de l'âme et de la meilleure façon d'y parvenir dans le cadre de l'économie domestique. Philodème suit expressément Métrodore et estime les différentes manières de gagner sa vie à l'aune du calcul des plaisirs : c'est ainsi que doivent être rejetés comme sources de revenu les conquêtes militaires, l'art équestre, l'exploitation des mines. L'homme vertueux, en revanche, s'il ne cultive pas luimême son jardin, peut retirer un revenu de terres qu'il fait cultiver. Néanmoins, la meilleure occupation pour un philosophe, c'est d'être dédommagé pour son enseignement philosophique. Ne peut-on pas voir là un écho à la pratique de Philodème lui-même auprès de Pison en Italie, sinon à Herculanum ? Il aurait sans doute été intéressant de poser en passant la question du philosopher à Rome, d'autant plus que les Romains sont nommés en col. XXV (ce qui est rarissime chez Philodème).

Deux notices sur le matériel papyrologique disponible (papyrus et dessins) et les éditions précédentes viennent clore cette riche introduction qui donne un bon aperçu du contenu en reprenant de façon méthodique les grands points traités par Philodème.

V. Tsouna reproduit, sans grands changements, l'édition de Jensen qu'elle traduit. L'apparat critique, sous forme de notes au texte, est réduit à sa plus simple expression, avec quelques maladresses, comme dans l'utilisation de l'abréviation «om. » p. 10, n. 6: Jensen n'a pas pu «omettre » un ajout textuel proposé par D. N. Sedley; idem pour les notes 18 et 25 (respectivement p. 40 et 50).

Signalons également certains choix textuels contestables. Ainsi, à la col. XV, 13,

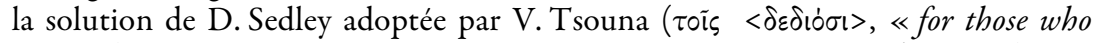
fear... ») est expliquée par une haplographie peu convaincante (note 44); on

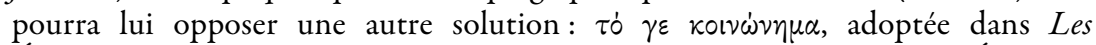
Épicuriens (p. 606) et justifiée par D. Delattre dans «Philodème, L'Économie (PHerc. 1424) : variantes textuelles d'un écrit plus que jamais d'actualité $\gg$, dans Rencontres papyrologiques en Bourgogne. Actes de la Journée d'étude (26 octobre 2011) en hommage à Patrice Cauderlier, Nancy, 2013, p. 65.

De même, la conjecture de D. N. Sedley en col. XVI, 30 ( $\dot{\varepsilon} \lambda \alpha \tau \tau \circ \tilde{\tau} \tau \alpha \iota$ $\tau \dot{\alpha})$ est absolument démentie par la photographie du papyrus et par le dessin qui confirment la lecture et la restitution de C. Jensen : ( $\dot{\varepsilon} \lambda \alpha \tau \tau \circ \tilde{v}[\sigma \theta] \alpha i \tau \varepsilon)$. Il ne faut pas comprendre «there is a diminution in the things that are conducive to the end... », mais plutôt : « ceux-ci sont assurément désavantagés pour ce qui regarde la fin... » (Les Épicuriens, p. 606). 
En outre, en col. XXI, 22 (n. 59), on peut clairement lire un $c h i$ sur le papyrus et la photographie (voir D. Delattre, art. cit. p. 47-67), ce qui rend caduc le choix éditorial de Jensen.

Signalons enfin que, dans le cas des col. XXII 48-XXIII 1, si V. Tsouna reproduit le texte conjecturé par Jensen, elle s'abstient curieusement de le traduire ; la traduction proposée dans le volume de la Pléiade (« et sont les plus sensés », p. 612) repose quant à elle sur la conjecture (omise sur le site du CISPE) : [ $\nu(\mu \omega \tau \dot{\alpha} \tau \omega] \nu$.

Vingt-trois pages de notes pertinentes, une bibliographie succincte et un utile index des mots grecs viennent clôturer ce livre. À noter cependant quelques erreurs ou oublis dans la bibliographie. Ainsi p. 103, on relèvera « Aristotle » (tel qu'il est cité par books.google.com) au lieu d'《Aristote ». Parmi les ouvrages relatifs à cette œuvre de Philodème, on déplorera (dans le premier tirage du moins) l'absence de référence aux Épicuriens, où elle est traduite et annotée par D. Delattre et... V. Tsouna elle-même.

Annick MONET

Yelena BARAZ, A Written Republic: Cicero's Philosophical Politics, Princeton, Princeton University Press, 2012, X+252 pages, ISBN 978-0-6911-5332-2.

Dans la foulée des publications récentes sur Cicéron philosophe, cette excellente étude explore à nouveaux frais son projet « encyclopédique » (sous la dictature de César, 46-44) par le biais d'un examen des prologues. Yelena Baraz (désormais Y.B.) justifie dans son introduction cette approche méthodologique. Cicéron possédait un cahier de prologues (volumen prohoemiorum) et écrivait des prologues indépendamment de l'ouvrage (ad Att. XVI, 6, 4). Cela signifie, explique-t-elle, qu'il concevait son projet comme un tout et qu'il était conscient qu'il faisait face aux mêmes types d'objections, auxquelles les prologues ont pour principale fonction de répondre, remplissant ainsi la fonction de captatio benevolentiae (p. 6-7). Les prologues constituent en cela des documents uniques révélant les intentions de l'auteur et les réserves de ses lecteurs ainsi que les tensions entre les prétentions philosophiques à l'universalité et les contraintes sociales (p. 8). Je résumerai d'abord le livre chapitre par chapitre et ferai ensuite quelques observations critiques.

Les deux premiers chapitres offrent une mise en contexte à la fois sociohistorique et personnelle. Au chapitre 1, Y.B. offre une analyse comparative des prologues de Salluste et du traité anonyme Rhetorica ad Herennium qui nous informe sur les préjugés du lectorat de l'époque, soit l'élite romaine, et sur les stratégies possibles pour les surmonter. Aux yeux de ce lectorat, l'activité d'écrivain doit se confiner à la sphère de l'otium, par opposition au travail (negotium) caractéristique de la vie politique (p. 17, 42; cf. Tusc. II, 1). Tandis que Salluste confronte directement la conception traditionnelle et défend l'écriture comme travail et condition de la mémoire collective et de la gloria (Cat. 1, p. 27, 31, 35), l'auteur anonyme se conforme au contraire à l'opinion majoritaire et dit confiner son activité intellectuelle à l'otium (p. $42 ; c f$. Varron, p. 39). Cicéron hésitera toujours, selon Y.B., entre ces deux stratégies, notamment en raison de son désir d'atteindre 